\title{
La refonte du fédéralisme suisse: impacts sur l'autonomie cantonale et communale
}

\author{
Laetitia Mathys et Nicolas Keuffer \\ Université de Lausanne (soumission spéciale)
}

\section{Introduction}

Il est souvent dit que la Suisse, Etat fédéral constitué en 1848, n'est pas un pays comme les autres, qu'elle est unique, complexe et intrigante à plus d'un niveau. Plus précisément, trois. Le pouvoir politique est en effet partagé entre la Confédération, les cantons et les communes ${ }^{1}$. Pour un pays comptant quatre régions linguistiques et autant de différences culturelles et géographiques, le fédéralisme est la garantie d'une cohésion sociale à terme (Ladner, 2013). La littérature sur le fédéralisme suisse est très étendue et fait état de deux principales dimensions fédérales dans lesquelles s'inscrivent la marge de manœuvre des différentes entités souveraines: les dimensions verticale et horizontale. La première implique une collaboration à tendance plus ou moins hiérarchique entre la Confédération, les cantons et les communes. La deuxième engage, elle, un ou plusieurs cantons ou communes qui collaborent de manière égale et non-hiérarchique, à l'aide, par exemple, de concordats, de conventions ou d'autres contrats. Cette étude considère essentiellement la dimension verticale, dont plusieurs principes fondamentaux forment le substrat du fédéralisme suisse: selon le principe de souveraineté inscrit à l'article 3 de la Constitution de 1999 tout d'abord, chaque niveau institutionnel possède sa propre législation, son gouvernement, son parlement (ou assemblée) et édicte ses propres lois qui doivent être compatibles avec les lois fédérales; ensuite, le principe de subsidiarité développé à l'article 5a prévoit l'accomplissement des tâches étatiques par le niveau le plus inférieur si celui-ci est réalisable et non expressément attribué à l'échelon supérieur, gage de décentralisation; par conséquent, enfin, les cantons et les communes possèdent une grande autonomie et indépendance à l'égard de l'Etat fédéral et connaissent différents moyens de participation aux processus décisionnels de l'échelon supérieur.

Si la Suisse est quelque peu singulière à de nombreux égards, elle n'est pas étrangère à certains blocages et dysfonctionnements internes relatifs à l'organisation des prestations étatiques, ayant légitimés la prise en considération de certains courants réformateurs du secteur public. Cette étude se construit autour de la plus importante réforme effectuée en Suisse (Vatter, 2006): la réforme de la répartition des tâches, votée en 2004 et mise en œuvre en 2008. Réorganisation fondamentale du fédéralisme, elle est surtout le produit de conflits divergents, de visions qui s'affrontent et de logiques qui se restructurent. Elle bouscule les rapports de pouvoirs entre la Confédération, les cantons et les communes, afin de revitaliser en profondeur le fédéralisme. Cet article s'intéresse donc, d'une part, à la manière dont la Suisse a fait évoluer son fédéralisme conformément à son contexte spécifique et, d'autre part, aux répercussions que ces évolutions ont eues sur l'autonomie des cantons et des communes. Pour ce faire, après avoir brièvement explicité la notion d'autonomie dans le cadre de l'approvisionnement des services publics, nous présentons la réforme de la répartition des tâches, sa genèse, ses spécificités, ses principes, ses influences et ses répercussions constitutionnelles. Ensuite, nous nous intéressons aux impacts de la réforme sur l'autonomie cantonale et communale respectivement. Nous mettons enfin les principales tendances en perspective, pour finalement en tirer des conclusions.

\footnotetext{
${ }^{1}$ Au premier janvier 2015, la Confédération est constituée de 26 cantons, états fédérés souverains et égaux, et de 2324
} communes. 


\section{La réforme de la répartition des tâches}

\section{L'autonomie liée à l'accomplissement des tâches publiques}

Légitimée par la Constitution, la notion d'autonomie peut directement être liée à la capacité pour une entité institutionnelle de définir les tâches à accomplir dans le cadre de sa compétence (art. 43) et, conformément à l'art. 47 al. 2, d'être reconnue juridiquement par l'entité supérieure : "La Confédération laisse aux cantons suffisamment de tâches propres et respecte leur autonomie d'organisation. Elle leur laisse des sources de financement suffisantes et contribue à ce qu'ils disposent des moyens financiers nécessaires pour accomplir leurs tâches ». De la souveraineté des 26 cantons qui constituent la Confédération helvétique relèvent les communes, institutions de droit cantonal qui forment l'échelon de base de l'organisation politique et territoriale suisse. Tous les cantons se composent de communes politiques et définissent ainsi leur organisation, leurs compétences et leurs ressources. Leur autonomie s'inscrit donc dans les limites fixées par le droit cantonal, comme en fait référence l'art. 50 de la Constitution fédérale ${ }^{2}$. II faut cependant souligner qu'en plus des tâches qui leur sont confiées par leur canton respectif ou par la Confédération, les communes déterminent leurs propres compétences dans plusieurs domaines relevant du niveau local en vertu de leurs compétences générales résiduelles et doivent être ainsi considérées comme des " collectivités publiques politiquement décentralisées » (Horber-Papazian et Jacot-Descombes, 2014, pp. 284-285).

Dans un Etat doté de structures fédérales comme la Suisse, la répartition des compétences et des tâches revêt une importance majeure, d'autant qu'elle implique une capacité décisionnelle, financière et opérationnelle plus ou moins étendue. Pour chaque domaine de tâches, il s'agit d'évaluer les avantages et inconvénients qui découleraient d'une centralisation ou, au contraire, d'une décentralisation, afin d'obtenir le degré de responsabilité optimal et la meilleure mise à disposition des prestations que possible. Le fédéralisme n'a donc nullement pour principe de décentraliser systématiquement tous les services publics, mais d'attribuer les différentes tâches aux échelons qui sont le mieux à même de les assumer (Wellstein, 2001).

\section{Une réforme aussi radicale qu'inédite}

Le 28 novembre 2004, le peuple suisse a adopté avec $64.4 \%$ des voix et 23 cantons pour une participation au vote de $36.85 \%$, la plus importante réorganisation des responsabilités institutionnelles en Suisse, allant même jusqu'à redynamiser le fédéralisme en profondeur, à savoir la réforme de la répartition des tâches et de la péréquation financière (RPT) ${ }^{3}$. Entrée en vigueur le $1^{\mathrm{er}}$ janvier 2008, cette réforme veut désenchevêtrer les compétences institutionnelles, donner plus de marge de manœuvre aux cantons et augmenter l'efficacité de la mise en œuvre des politiques publiques (Dafflon, 2004). Elle bouscule la tradition conservatrice de la Suisse puisqu'elle est la première depuis 1848 à vouloir remodeler le fédéralisme et restructurer les rapports institutionnels en privilégiant deux principes : la subsidiarité et l'équivalence fiscale. Dans ce dernier cas, il s'agit de la possibilité donnée aux bénéficiaires d'une prestation étatique de décider de cette prestation et d'en supporter également les coûts. La réforme est considérée comme la solution pour pallier les nombreuses difficultés qui se sont graduellement déclarées depuis les années 50 et surtout pour redonner du pouvoir aux cantons.

Pour mieux comprendre l'origine de cette réforme, il est nécessaire d'en présenter les grandes lignes. Déjà pendant la période des Trente Glorieuses (40-70) et avec l'effet de la globalisation, le nombre des tâches publiques augmente, ainsi que les exigences de la population quant à la qualité du service public (Jacot-

\footnotetext{
${ }^{2}$ Art. 50 : « 1. L'autonomie communale est garantie dans les limites fixées par le droit cantonal. 2. La Confédération tient compte des conséquences éventuelles de son activité pour les communes. 3. Ce faisant, elle prend en considération la situation particulière des villes, des agglomérations urbaines et des régions de montagne. » Par ailleurs, il convient d'ajouter que les constitutions cantonales garantissent souvent et explicitement l'autonomie communale.

${ }^{3}$ Dans cet article, il est principalement question du premier axe de la RPT : la répartition des tâches.
} 
Descombes, 2013). Dans le cadre de la relation entre la Confédération et les cantons, ces derniers, ne pouvant pas assumer la mise en œuvre ni les coûts de toutes ces nouvelles tâches publiques, donnent progressivement et volontairement des compétences à la Confédération qui se charge de lourds domaines publics tels que l'environnement, la prévoyance sociale ou encore l'aide au développement (Freiburghaus, 2005). Cependant, au fil du temps, la balance entre les dépenses et les recettes fédérales n'augmente pas aux mêmes proportions, ce qui oblige la Confédération à prendre de drastiques mesures d'économie. En 1980, le peuple suisse vote favorablement pour le "Programme d'économies 1980 ", qui prévoit de diminuer ou de supprimer des quotes-parts des cantons sur certaines taxes ou même à diminuer de $10 \%$ les subventions fédérales versées aux cantons ${ }^{4}$. Ces pressions financières et de mise en œuvre de la Confédération sur les cantons s'ajoutent aux problèmes toujours croissants d'enchevêtrement des tâches et des responsabilités dans l'application des politiques publiques. Les cantons perdent graduellement leur autonomie et leurs compétences décisionnelles, en plus des difficultés financières et humaines dans la mise en œuvre des politiques publiques auxquelles ils font face.

Des tendances similaires se dessinent à la même période au niveau de la relation entre les cantons et les communes. A l'échelon inférieur, en effet, les communes suisses sont confrontées à un certain nombre de problèmes et enjeux émergeant dès la deuxième partie des années 90 , tels que la complexification et la multiplication des tâches et des charges qui leur incombent quand bien même leurs capacités d'action et leur autonomie diminuent (Geser, 1997), l'exigence de plus en plus forte des citoyens ${ }^{5}$ vis-à-vis de l'efficience et de l'efficacité de l'action publique, la forte imbrication des compétences d'exécution, de décision et de financement, ainsi que le manque de coordination entre les communes et "leur " canton (Ladner et al., 2000), ou encore la remise en question de l'espace institutionnel communal (Leresche 1996). C'est dans un contexte caractérisé par la persistance - voire même l'intensification - de ces difficultés (Steiner et Ladner, 2006) que la réforme de la répartition des tâches s'inscrit au niveau local. Tout comme pour l'échelon supérieur, elle se fonde sur des critères tant économiques que politiques - les principes du décideur-payeur, de la subsidiarité, de la neutralité des coûts et de la qualité des prestations fournies - et vise à renforcer la transparence et l'efficacité des collaborations institutionnelles verticales et ainsi à concourir au renouvellement du fédéralisme local.

\section{Influence du modèle de la Nouvelle Gestion Publiques (NGP)}

Ce sont dès les années 80 que l'administration publique suisse a été la cible d'une nouvelle rationalité de gestion publique, mais également d'une nouvelle légitimité basée sur l'efficience et la qualité des prestations fournies et des dépenses économes (Emery, 2000). Les problèmes financiers de la Confédération dès la fin des années 70 , les nombreux doublons administratifs et la lenteur des procédures révélaient une faille de gestion publique. Cette dernière était condamnée à se moderniser. Les objectifs des différentes réformes qui ont eu lieu dans l'administration publique suisse peuvent se résumer en trois modèles (Giauque et Emery, 2008) :

1. Le modèle de l'efficience, visant à rendre les organismes publics plus efficients et efficaces en prenant les outils de gestion des entreprises privées. En termes d'efficacité, les objectifs sont définis et doivent être atteints dans une limite de temps. L'efficience concerne l'utilisation rationnelle des ressources, le fonctionnement au moindre coût. Pour ce faire, l'administration devait évoluer d'une gestion basée sur ses ressources (inputs) à une administration gérée par prestations (outputs).

\footnotetext{
${ }^{4}$ Les 3 modifications constitutionnelles suite à la votation du Programme d'économies 1980 sont les suivantes : 1.

Suppression de la quote-part des cantons au produit net des droits de timbres 2. Réduction de la quote-part des cantons au bénéfice net de la Régie des alcools 3. Suppression de la subvention fédérale destinée à abaisser le prix du pain (Explication du Conseil fédéral de la votation populaire du 30 novembre 1980).

${ }^{5}$ Afin de ne pas alourdir le texte, nous avons renoncé à une rédaction systématiquement épicène. Toutefois, chaque fois que cela se justifie, un terme écrit au masculin s'entend aussi au féminin et vice versa
} 
2. Le modèle de la décentralisation et de la flexibilité, voulant séparer l'opérationnel de la gestion stratégique, redistribuer les compétences décisionnelles aux niveaux les plus proches de la clientèle ou du terrain avec davantage de souplesse. Pour ce faire, le recours à des contrats de prestations est nécessaire.

3. Le modèle de la qualité, prévoyant de favoriser la formation continue et de recourir à des enquêtes pour mesurer la satisfaction des « clients » du service public.

Comme toutes les réformes qui ont eu lieu depuis les années 80 dans l'administration publique suisse, la réforme de la répartition des tâches emprunte ces modèles et s'inscrit au cœur du phénomène de la NGP (Cappelletti et al., 2014). Si l'on se réfère aux arguments mis en exergue en 2008 par le Département fédéral des finances (DFF), elle permettrait d'améliorer l'efficacité et l'économicité des affaires publiques, tant au niveau du gouvernement que de l'administration. Elle éviterait ainsi le gaspillage des deniers publics en ciblant mieux les dépenses et en supprimant les incitations inopportunes en faveur de projets dispendieux. Enfin, elle use de moyens tirés directement du secteur privé puisqu'elle prévoit l'utilisation de contrats publics basée sur des objectifs et des indicateurs et recourt ponctuellement aux enquêtes de satisfaction, aux contrôles et aux évaluations afin de garantir la qualité de la gestion publique (Athias, 2013).

\section{Une refonte constitutionnelle}

L'importance de la réforme de la répartition des tâches en Suisse se traduit par la modification de 27 articles de la Constitution de 1999 qui en compte au total 196 (Fleiner, 2002). Elle oblige l'Etat, les cantons et les communes à optimiser leurs prestations et leurs responsabilités dans l'implémentation des politiques publiques. Cette refonte constitutionnelle représente un changement structurel et inédit de la manière de procéder. Elle bouleverse l'idée conservatrice selon laquelle le système helvétique est figé et rigide : " alors que l'Allemagne et l'Autriche, de leur propre aveu, ont eu beaucoup de peine à introduire des réformes minimes dans leur système fédéral, les électeurs et électrices suisses ont adopté la réforme en 2004 par scrutin populaire» (Frey et al., 2005). Bien au contraire, cette réforme fait preuve de dynamisme et révèle une forte volonté de changement de la part des politiciens et des citoyens. Dans les années 60, le fédéralisme dit d'exécution (la Confédération décide - les cantons exécutent) était fortement critiqué car les cantons s'apparentaient davantage à des soldats soumis aux ordres des directives fédérales (Knapp, 1986 ; Wälti, 2007). Les médias parlaient même de crise de mise en œuvre suite aux non-respects récurrents des délais de la part des cantons et de leurs difficultés de financement. La réforme prévoyait de redéfinir le fédéralisme en allant vers un fédéralisme dual (claire séparation des compétences de mise en œuvre et fiscales entre les institutions) qui avait pour ambition d'augmenter radicalement le degré d'autonomie des cantons. La situation actuelle ne ressemble pourtant pas à ce modèle dualiste puisque les compétences exécutives et financières restent largement partagées. Il est aujourd'hui plutôt question d'un fédéralisme coopératif qui se renforce autour d'une gestion commune de tâches publiques. Suite à la réforme, dix tâches sont passées exclusivement sous responsabilité cantonale alors que sept tâches ont été attribuées à la Confédération. Cependant, il n'a pas été possible de diviser les compétences dans le cas de vingt-et-une tâches publiques. Ces tâches restantes, dites "communes", sont traitées par des contrats de droit public passés entre la Confédération et les cantons: les conventions-programmes. Cet instrument repose sur l'art. 46 al. 2 de la Constitution qui prévoit la participation de la Confédération et des cantons dans la mise en place de programmes destinés à l'accomplissement des tâches publiques. Valables pour des périodes de 4 ans (200811, 2012-15, 2016-19), les conventions-programmes permettent de passer d'un système de subventions spécifiques basées sur l'avancement et les coûts des travaux (inputs) à des subventions globales ou forfaitaires planifiées pour la période quadriennale (outputs). Elles conduisent la Confédération et les cantons à discuter des montants et des objectifs contractuels dans le cadre d'un processus de négociation. La collaboration verticale doit désormais tendre vers un partenariat non-hiérarchique et doit permettre 
davantage d'autonomie aux cantons ${ }^{6}$. Plus encore, ces contrats poussent les cantons à contacter des prestataires de services tels que des associations ou des entreprises privées pour la réalisation de la mise en œuvre, ainsi qu'à consulter les communes. Les conventions-programmes, produits directs de la réforme de la répartition des tâches, appliquent donc strictement les modèles de la NGP. L'ambition prônée est qu'une véritable gouvernance multi-niveaux s'établisse entre les trois niveaux institutionnels grâce à cette nouvelle forme de collaboration institutionnelle dans laquelle il est plutôt question de procédures de coopération nonhiérarchiques entre les acteurs (Hooghe et Marks, 2001).

Puisque ce passage d'un fédéralisme d'exécution à un fédéralisme réellement coopératif implique également le niveau inférieur de l'organisation territoriale et politique suisse, un article constitutionnel a été intégré pour reconnaître le rôle consultatif des communes en sus de celui d'agent de mise en œuvre. L'art. 50 de la Constitution, introduit en 1998, récompense les efforts de l'Association des communes suisses (ACS) et de I'Union des villes suisses (UVS) pour que soit reconnu l'échelon local dans la structure étatique suisse, dans la mesure où il « ancre et précise le statut des communes dans l'Etat fédéral ; [...] cette disposition doit être comprise avant tout comme une incitation à la coopération verticale. [...] En ce sens, [elle] s'inscrit dans le renforcement d'un fédéralisme participatif qui a largement inspiré la mise à jour de la Constitution fédérale et dont les cantons aussi ont bénéficié. " ${ }^{7} \mathrm{Si}$ le Conseil fédéral accorde une attention particulière aux cantons, $c^{\prime}$ est que pendant de nombreuses années, ces derniers se sont montrés réticents à l'endroit d'un texte visant à rapprocher les exigences citoyennes avec les mesures prises pour répondre à celles-ci, mais qui, selon eux, amoindrissait en même temps leur souveraineté : la Charte européenne de l'autonomie locale (Conseil de l'Europe, 1985). Elaboré en 1985 par le Conseil de l'Europe dans le but de protéger et renforcer l'autonomie locale en Europe, ce texte, ayant qualité de traité international, n'a été ratifié qu'en 2004 par la Suisse. Les réserves des cantons s'expliquent en grande partie par les spécificités du système suisse, à savoir plus précisément son système de milice, le fait que les compétences matérielles sont définies par le droit cantonal et enfin par la prépondérance des subventions liées à des projets spécifiques vis-à-vis des subventions à vocation générale. Quoi qu'il en soit, ces deux textes juridiques - l'article 50 et la Charte - ont pour ambition de contribuer à assoir l'importance du niveau communal dans la collaboration intergouvernementale suisse (Politikverflechtung) s'opérant dans la délivrance des services publics (Scharpf, 1978), et devraient conduire à l'avenir à un renforcement des relations entre la Confédération et les communes (Meyer, 2010). La création de la Conférence tripartite sur les agglomérations (CTA), plateforme de dialogue permettant de prendre en considération la situation particulière des villes, des agglomérations urbaines et des communes, illustre par exemple une de leurs premières répercussions.

\section{Autonomie cantonale}

Les 26 cantons qui forment la Suisse sont souverains en tant que leur souveraineté n'est pas limitée par la Constitution fédérale et exercent tous les droits qui ne sont pas délégués à la Confédération. Chaque canton possède ainsi sa propre constitution, son gouvernement, son parlement (ou assemblée) et édicte ses propres lois, qui doivent être en adéquation avec le droit fédéral. Les cantons possèdent donc une grande autonomie et indépendance à l'égard de l'Etat fédéral. Ils ont de grandes compétences en matière d'éducation, d'environnement, de culture, de santé et dans d'autres domaines non-spécifiés dans la Constitution qui peuvent être fédérales ou conjointes : ce sont les « compétences résiduelles » (Ladner, 2013). Par exemple,

\footnotetext{
${ }^{6}$ L'art. 46 al. 3 de la Constitution fédérale le dicte : « La Confédération laisse aux cantons une marge de manœuvre aussi large que possible en tenant compte de leurs particularités ".

${ }^{7}$ Réponse du Conseil fédéral du 13.09.2000 à I'Interpellation 00.3404 déposée par Andreas Gross le 23.06.2000 au Conseil national « Interprétation de l'article 50 de la Constitution fédérale.

http://www.parlament.ch/f/suche/pages/geschaefte.aspx?gesch_id=20003404\# (consulté en janvier 2015).
} 
l'immigration ou l'asile sont des compétences fédérales mais la citoyenneté a toujours été avant tout communale. Ils sont également très autonomes dans leurs systèmes de taxation, dans leurs procédures budgétaires et dans leurs modes d'élections. Cette marge de manœuvre est fondamentalement grande pour les cantons, ce qui créé des disparités importantes, rendant parfois une uniformisation des procédures impossible. Au niveau législatif, les cantons ont un pouvoir de veto contre les lois fédérales et peuvent également affecter la législation fédérale à travers des initiatives cantonales. Des représentants cantonaux sont encore impliqués dans plusieurs commissions fédérales et consultés. Enfin, les cantons ont créé la Conférence des gouvernements cantonaux (CdC) en 1993 pour se donner les moyens de préserver leur autonomie et avoir une assise importante dans les décisions qui se prennent à Berne.

L'autonomie cantonale en Suisse se révèle être très élevée en comparaison internationale, comme l'ont démontré Hooghe, Liesbet, Gary Marks, Arjan H. Schakel en 2010. Ces auteurs ont calculé le degré d'autonomie régionale dans 43 pays démocratiques entre 1950 à 2011 et ce, sur la base de 9 indicateurs, pour obtenir le Regional Autonomy Index (RAI). Nous avons traduit la description donnée pour la Suisse selon les différentes dimensions imaginées pour l'étude (Tableau 1). Les indicateurs sont divisés entre deux catégories: les règles exclusives (self-rules) et les règles communes (shared-rules). Les règles exclusives sont appliquées uniquement par l'autorité et se portent sur l'autonomie fiscale, la portée politique et le système de représentation, alors que les règles communes sont exécutées conjointement par différentes autorités et concernent l'élaboration des lois, les contrôles institutionnel et fiscal et les réformes constitutionnelles. L'application de ces deux types de règles est destinée à coordonner les acteurs étatiques de sorte à poursuivre un but commun tout en préservant l'intégration de chaque acteur. Les résultats de l'étude de Hooghe et al. (2010) révèlent que l'indice le plus élevé calculé en fonction des critères de "Self-rule " est attribué à la Suisse. Le RAI montre que l'autonomie régionale en Suisse est parmi les plus élevées, après I'Allemagne et le Canada.

\section{Tableau 1: portrait de l'autonomie cantonale suisse et le codage attribué par les auteurs de l'étude}

\begin{tabular}{|c|c|c|}
\hline Indicateurs & Description & Code \\
\hline \multicolumn{3}{|c|}{ Self-Rule } \\
\hline $\begin{array}{l}\text { Institutional Depth } \\
\text { and Policy Scope }\end{array}$ & $\begin{array}{l}\text { Grandes compétences en matière d'éducation, d'environnement, } \\
\text { de culture, de santé, dans le gouvernement local et autres } \\
\text { compétences résiduelles. }\end{array}$ & $\begin{array}{l}\text { Depth: } 3 \\
\text { Scope: } 4\end{array}$ \\
\hline Fiscal Autonomy & $\begin{array}{l}\text { Les cantons sont libres de structurer et former leur système de } \\
\text { taxation. }\end{array}$ & 4 \\
\hline Borrowing Autonomy & $\begin{array}{l}\text { Parce que les gouvernements cantonaux sont par principe } \\
\text { autonomes et indépendants dans leurs procédures budgétaires, ils } \\
\text { peuvent emprunter au gouvernement fédéral sans aucunes } \\
\text { restrictions (Conseil de l’Europe, 1997) }\end{array}$ & 3 \\
\hline Representation & $\begin{array}{l}\text { Les élections populaires pour les parlements cantonaux prennent } \\
\text { place tous les } 4 \text { ans et chaque canton a son propre cycle électoral. }\end{array}$ & $\begin{array}{l}\text { Assembly : } 2 \\
\text { Executive : } 2\end{array}$ \\
\hline \multicolumn{3}{|c|}{ Shared Rule } \\
\hline Law Making & $\begin{array}{l}\text { Chaque canton a deux représentants et chaque demi-canton en a } \\
\text { un dans la Chambre haute, soit le Conseil des Etats. Ils ont un } \\
\text { pouvoir de veto sur les lois fédérales et les cantons peuvent } \\
\text { également affecter la législation fédérale à travers des initiatives } \\
\text { cantonales. }\end{array}$ & $\begin{array}{r}0.5 \\
0 \\
0.5 \\
0.5\end{array}$ \\
\hline Executive Control & $\begin{array}{l}\text { Consultation des cantons pour formuler et mettre en œuvre des } \\
\text { politiques publiques. Ils sont impliqués dans les commissions } \\
\text { fédérales et consultés durant le processus législatif. Cependant, le } \\
\text { Conseil fédéral n'invite pas les cantons à participer, ni à suivre } \\
\text { leurs conseils. La constitution de } 1999 \text { établit toutefois le droit aux } \\
\text { cantons de participer aux décisions de politique étrangère. Au fil }\end{array}$ & 1 \\
\hline
\end{tabular}




\begin{tabular}{|l|l|c|}
\hline & $\begin{array}{l}\text { du temps, des concordats se sont mis en place ainsi que 16 } \\
\text { conférences des directeurs cantonaux pour renforcer la } \\
\text { participation cantonale dans les décisions de politiques publiques. }\end{array}$ & \\
\hline Fiscal Control & $\begin{array}{l}\text { Ils influencent les décisions fédérales sur la distribution des taxes } \\
\text { sur les revenus. }\end{array}$ & 1 \\
\hline Borrowing Control & $\begin{array}{l}\text { La conférence des gouvernements cantonaux mise en œuvre en } \\
1993 \text { pour coordonner la politique étrangère et européenne a } \\
\text { progressivement évolué vers une conférence intercantonale qui } \\
\text { coordonne les transactions, mais les emprunts en sont exclus. }\end{array}$ & 0 \\
\hline $\begin{array}{l}\text { Constitutional } \\
\text { Reform }\end{array}$ & $\begin{array}{l}\text { Un changement constitutionnel demande un référendum } \\
\text { remporté par la double majorité des cantons et des citoyens. }\end{array}$ & 1 \\
\hline
\end{tabular}

Source : adapté de Hooghe et al., 2010. Le codebook qu'utilisent les auteurs est élaboré avec un codage prédéfini, noté entre 0 (absence d'effet) à 4 (présence d'effet) qui représente une échelle d'intensité

Figure 1: Regional Autonomy Index (RAI)

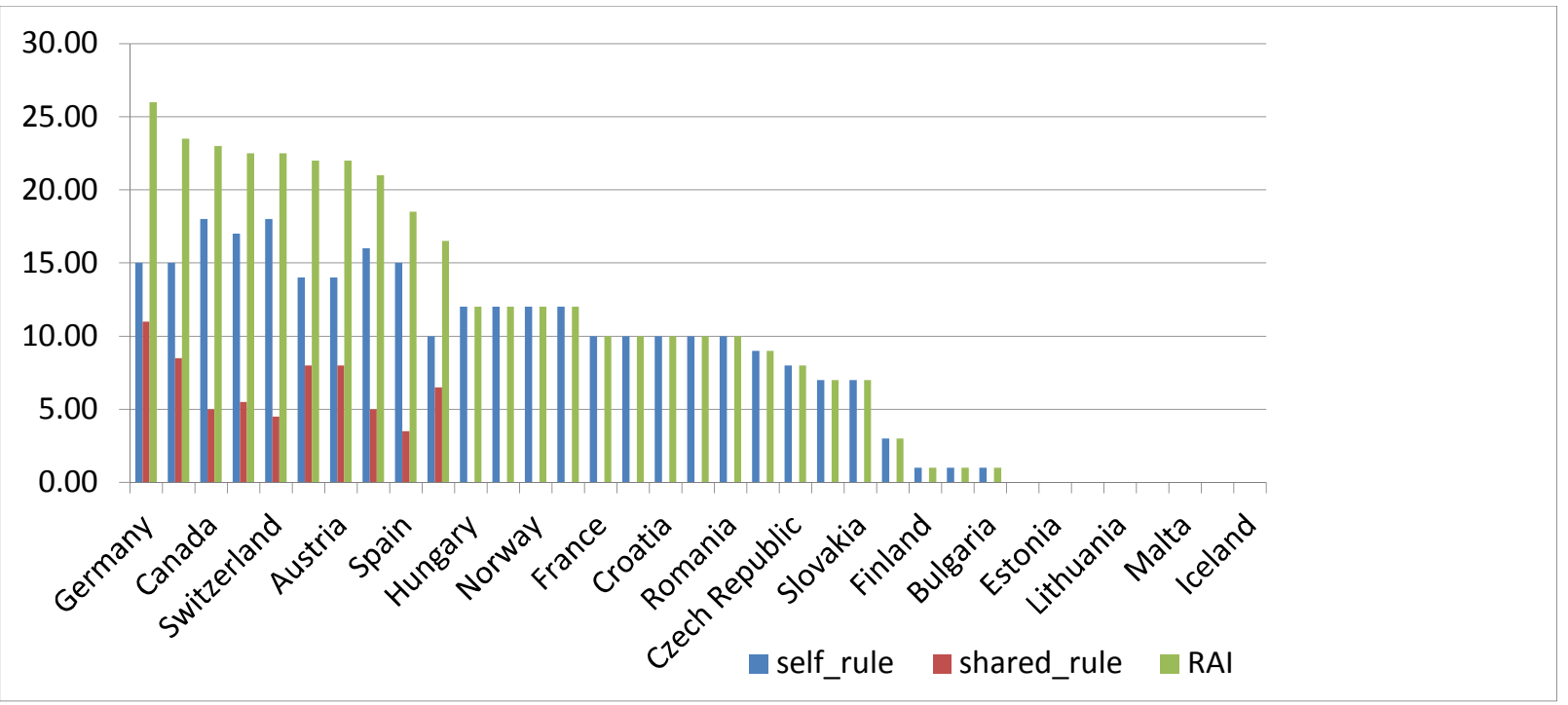

Source : adapté de Hooghe et al., 2010

Suite à la réforme et bien que l'autonomie régionale était déjà élevée en comparaison internationale (Figure 1), les cantons ont acquis davantage de marge de manœuvre dans l'élaboration et la mise en œuvre des politiques. L'introduction des conventions-programmes y joue un rôle majeur. En effet, le système de financement basé désormais sur des subventions forfaitaires ou globales confère davantage de marge de manœuvre aux cantons puisque ceux-ci peuvent gérer leurs dépenses de façon indépendante durant toute la période du contrat, c'est-à-dire quatre ans (Figure 2). De plus, les conventions-programmes impliquent un processus de négociation durant lequel les cantons discutent des montants des subventions et échangent leurs points de vue avec les responsables fédéraux. Par ce biais, les cantons peuvent davantage participer et prendre la parole. En plus d'une officialisation du rôle stratégique de la Confédération, les cantons se voient formellement attribuer un rôle opérationnel dans la mise en œuvre et le financement des politiques publiques, contrairement à la situation qui existait avant la réforme. En effet, les cantons devaient rendre des comptes à la Confédération qui finançait les démarches au cas par cas et avait un avis prononcé sur l'ensemble des opérations. Alors que désormais, les cantons gèrent directement le coût et les délais d'application des tâches sur le terrain, même si la Confédération s'autorise des contrôles pour assurer un suivi régulier. Avant la réforme, les cantons, possédant de nombreux pouvoirs résiduels, prenaient pour acquis la mise en œuvre de certaines politiques publiques, alors que celles-ci étaient déjà appliquées et gérées par la Confédération. Cette situation engendrait des doublons de compétences, des enchevêtrements opaques et des confusions malheureuses (Armingeon, 2000). Par conséquent, en plus d'une séparation de compétences, 
le rôle stratégique donné à la Confédération permet d'orienter les procédures et éviter que cette situation ne réapparaisse.

\section{Figure 2 : les trois objectifs principaux de la réforme}

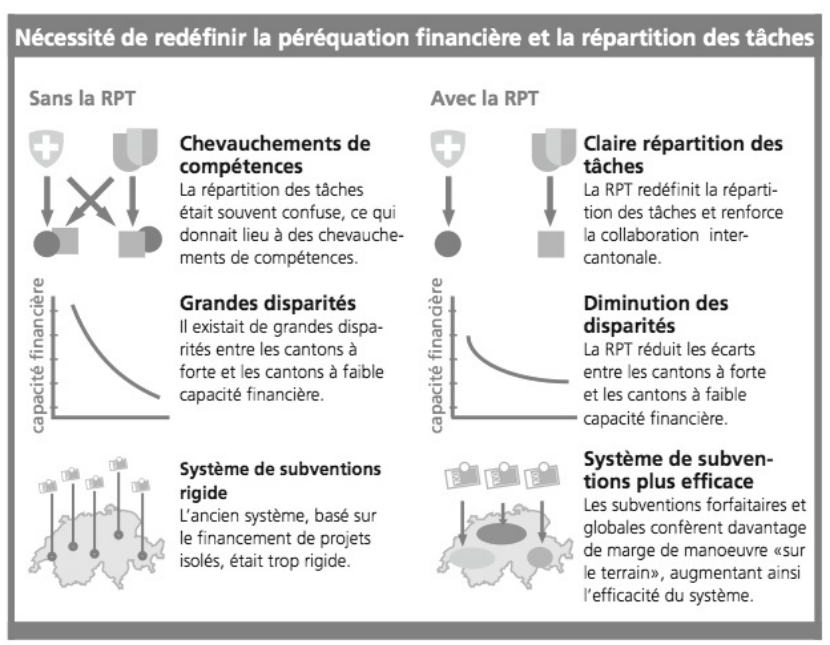

Source : CdC, "La RPT entre la Confédération et les cantons », septembre 2007

\section{Limites à l'autonomie cantonale}

En juin 2014, la Fondation pour la collaboration confédérale a publié un rapport intitulé " Monitoring du fédéralisme 2011-2013 ". II reporte l'analyse réalisée par les cantons et les conférences intercantonales sur l'évolution du fédéralisme en Suisse. Les cantons y évoquent leur satisfaction quant à leur collaboration avec la Confédération, notamment concernant le domaine de la politique d'intégration ${ }^{8}$ : " la Confédération et les cantons ont pu s'accorder dans le cadre d'un processus de négociation sur des objectifs stratégiques communs et sur une clé de répartition du financement $1: 1$ dans le domaine de l'intégration. » (Condensé, p. 4). De plus, le pilotage des tâches communes via les conventions-programmes leur laisse, selon eux, une grande marge de manœuvre. Ils félicitent également cette nouvelle collaboration verticale basée sur un partenariat. Malgré tout, il existe un risque que la Confédération ne reporte des charges sur les cantons suite à la prise de mesures d'économies (comme cela s'est produit en 1980) et le risque d'avoir de nouveaux enchevêtrements qui apparaissent, ce qui est clairement en contradiction avec les principes de la réforme. C'est le cas par exemple, du financement mixte des infrastructures ferroviaires. Avec le système des conventions-programmes, force est de constater qu'il existe toujours des problèmes de court-terme qui peuvent être assimilés à l'ensemble de la réforme de la répartition des tâches. Ceux-ci sont les suivants :

1. Une influence trop grande de la Confédération lors de la définition des objectifs (consignes toujours plus strictes);

2. Un manque de ressources financières et de personnel des cantons, qui finalement, négligent leurs propres domaines de compétences ;

3. Un manque d'orientation stratégique de la part de la Confédération dans certains domaines publics ;

4. Une faible communication de la Confédération avec les cantons.

Dans d'autres domaines politiques, les cantons dénoncent une centralisation pressante qui limite la souveraineté des cantons, comme dans le domaine de l'aménagement du territoire. Le Conseil fédéral a en outre changé le cap de sa politique énergétique " Stratégie énergétique 2050 » sans associer les cantons, bien que leurs intérêts et leurs compétences soient touchés en tout point de vue. Ces dernières années, la marge de manœuvre s'est vraisemblablement restreinte pour les cantons qui ont dû faire face à une pression

\footnotetext{
${ }^{8}$ La Convention-programme pour l'encouragement spécifique à l'intégration cantonale des étrangers est par exemple entrée en vigueur le 1er janvier 2014.
} 
toujours plus grande et certains décisions de la Confédération n'y sont pas étrangères : "les cantons sont encore et toujours confrontés à un report de charges et à une augmentation des charges financières et en personnel liée à la mise en œuvre du droit fédéral. » (p.5). II leur manque parfois des ressources financières et de la main d'œuvre pour l'accomplissement de tâches qui relèvent de leurs compétences. De plus, il est nécessaire d'associer encore plus les cantons aux processus de décisions de la Confédération et surtout en politique européenne car ceux-ci sont directement touchés par les mesures prises à ce niveau-là.

\section{Autonomie communale}

La Charte européenne de l'autonomie locale définit non seulement ce concept, par « le droit et la capacité effective pour les collectivités locales de régler et de gérer, dans le cadre de la loi, sous leur propre responsabilité et au profit de leurs populations, une part importance des affaires publiques " (Conseil de l'Europe $1985: 3$, al. 1), mais propose également un certain nombre de critères pour apprécier tangiblement son étendue ${ }^{9}$. L'autonomie locale est donc d'une part encouragée normativement en tant que pierre angulaire d'une démocratie véritable capable de répondre aux exigences des citoyens et de contrebalancer le pouvoir des gouvernements situés aux échelons supérieurs et, d'autre part, elle est constituée de multiples facettes, ce qui la rend complexe à appréhender (Verhoest et al, 2004 ; Clark, 1984). Ainsi, la littérature scientifique ne s'accorde pas sur les dimensions objectives qui doivent être prises en compte pour mesurer empiriquement les différents degrés d'autonomie locale (Hansen et Klausen, 2002). On peut évoquer ici brièvement la distinction effectuée entre les aspects économiques et sociaux liés intrinsèquement au contexte local et les caractéristiques de la relation du gouvernement local avec ceux du ou des niveaux supérieurs (Gurr et King, 1987). Dans ce dernier cas de figure, il est aisé de relever les dimensions liées respectivement à la situation juridique, aux moyens - particulièrement financiers - à disposition des gouvernements locaux, leur marge de manœuvre décisionnelle (Page et Goldsmith, 1987), leurs leviers d'influence politique (Page, 1991), ou encore la perception subjective des acteurs concernés (Ladner, 1994), puisqu'elles ont servi de fondements au développement de diverses célèbres typologies (Goldsmith et Page, 2010 ; Hesse et Sharpe, 1991).

En Suisse, il est plus usuel d'utiliser le terme d'autonomie communale que celui d'autonomie locale (Conseil fédéral, 2003, p. 72). Les communes suisses sont actuellement au nombre de 2352 (OFS, 2014) et se caractérisent par une extrême diversité, non seulement en termes de taille, de structures socio-économiques et de nombre de communes existant au sein d'un même canton, mais également, puisqu'elles dépendent respectivement des lois cantonales, dans l'étendue de leur autonomie (Horber-Papazian, 2006). Une étude comparative récente, appliquant 7 dimensions de l'autonomie communale aux 26 cantons suisses ${ }^{10}$, a permis de mettre en exergue la primauté de la culture comme variable explicative de la différence d'autonomie locale entre les cantons, la Suisse-allemande étant clairement caractérisée par un degré d'autonomie plus élevée que la Suisse-romande (Ladner et Keuffer 2014 ; Figure 2). Il est important de préciser néanmoins que de manière globale, les communes suisses bénéficient d'un degré d'autonomie élevé ${ }^{11}$, se concrétisant

\footnotetext{
${ }^{9}$ On peut ainsi mentionner la mesure selon laquelle le principe d'autonomie locale est ancré constitutionnellement et légalement (art. 2), l'étendue des compétences attribuées aux autorités les plus proches des citoyens (art. 4), la valeur de la consultation des collectivités locales dans le cadre du processus de décision (art. 4), ainsi que la portée de leurs capacités d'influence et des dispositions les protégeant (art. 5, 8 et 11), et enfin l'importance des ressources financières, administratives, fiscales et humaines dont elles disposent effectivement (art. 6 et 9).

${ }^{10}$ Les sept dimensions prises en compte sont les suivantes : Legal autonomy, Policy autonomy, Political autonomy, Fiscal autonomy, Social autonomy, Administrative autonomy et Vertical Influence (Ladner et Keuffer, 2014).

${ }^{11}$ Une étude mandatée par la Commission européenne intitulée "Self-rule Index for local authorities in the EU 19902014" consistant à mesurer le degré d'autonomie locale des pays européens en s'inspirant de Hooghe et al. (2010) a été confiée en octobre 2014 au Prof. Ladner (Institut de Hautes études en administration publique, Université de Lausanne, Suisse) et est en cours au moment de la rédaction du présent article. Les premiers résultats qui s’en dégagent confirment
} 
notamment par leur latitude unique au monde pour fixer de nouveaux impôts locaux et le taux auxquels les contributeurs sont assujettis (Ladner 2005).

Figure 2 : Degré total d'autonomie locale des différents cantons suisses (en rouge : les cantons francophones et italophones)

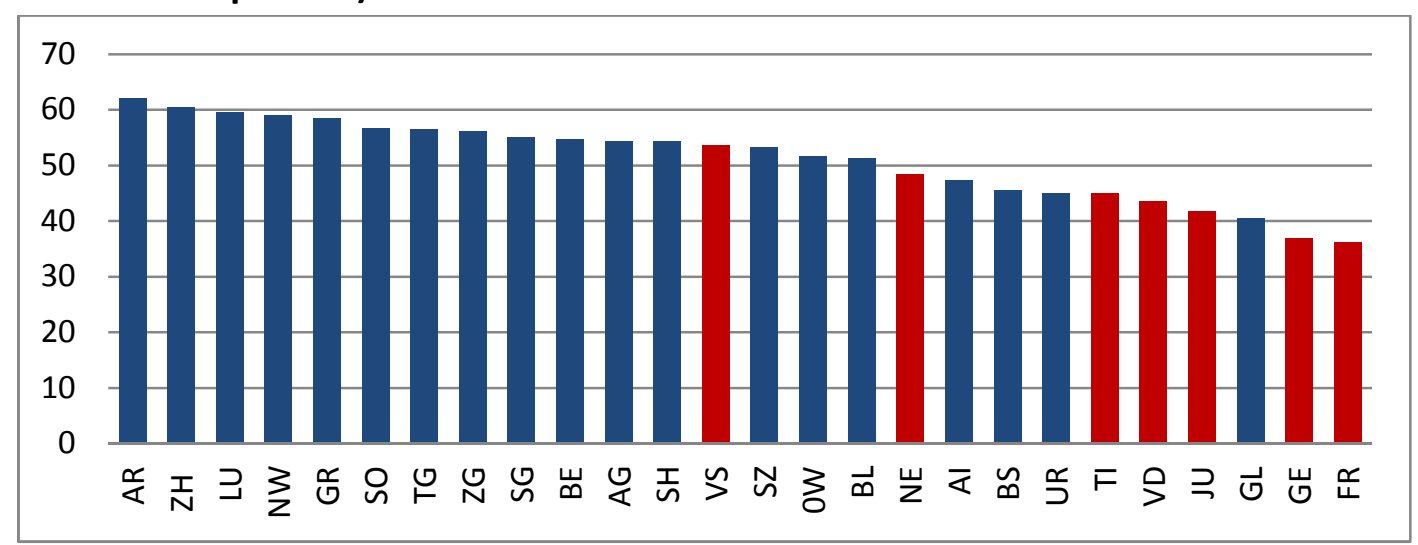

Source : adapté de Ladner et Keuffer, 2014

Il est difficile de rendre compte empiriquement des conséquences des réformes effectuées. D'une part, parce que la réforme de la répartition des tâches a été introduite au niveau fédéral et n'a qu'indirectement incité les cantons à entreprendre des réformes de leur propre répartition des tâches avec les communes. L'architecture fédéraliste suisse exigerait donc d'appréhender le régime de répartition des tâches entre les communes de chaque canton respectivement avant et après les réformes engagées afin d'en déceler les modifications. La proportion et le type de tâches attribuées par le canton par rapport à celles étant choisies par les communes varie en effet très fortement d'un canton à un autre. D'autre part, parce que les données récoltées auprès des communes suisses dans une ambition d'exhaustivité manquent. Le dernier questionnaire ayant été adressé à l'ensemble des communes, qui date de $2009^{12}$, met clairement en évidence le fait que les secrétaires communaux estiment globalement que l'autonomie de leur propre commune a diminué au cours des dix dernières années. La perception de cette chute est généralisée mais est encore davantage prégnante dans les petites communes (Figure 3). Les villes profitent effectivement désormais des politiques d'agglomération et du système de compensation des charges, prévus à l'art. 50 de la nouvelle Constitution fédérale.

Bien que les évolutions prévues n'avaient certainement pas encore produit tous leurs effets, les réponses des secrétaires communaux relatives aux effets des réformes ${ }^{13}$ tracent une tendance relativement claire: ils estiment que leur commune dispose de davantage de compétences de mise en œuvre, mais moins de capacités de financement et de décision. En ce qui concerne la formation au niveau local (l'école primaire), par exemple, plus de la moitié des secrétaires ont jugé que les réformes de répartition des tâches entreprises avaient engendré lors de ces dix dernières années une décentralisation des compétences opérationnelles

le cas particulier de la Suisse (Sonderfall) en ce sens que l'index d'autonomie locale est parmi les plus élevés avec ceux des pays nordiques.

${ }^{12}$ Ce 5ème questionnaire adressé aux secrétaires municipaux de toutes les communes suisses a été conduit par l'IDHEAP (Université de Lausanne) et le KPM (Kompetenzzentrum für Public Management - Université de Bern) et a un taux de réponse de $57.7 \%$. Sauf indication contraire, les résultats présentés ci-après en proviennent.

${ }^{13}$ Question 20 : « Dans quels domaines y a-t-il eu, au cours des dix dernières années, un transfert de charges financières, de tâches à accomplir et/ou de compétences décisionnelles entre la commune et le canton ? " Question 22 : " De manière globale, avez-vous constaté les effets suivants suite aux transferts de charges/tâches et de compétences décisionnelles ? "; Question 18b : " Le degré d'autonomie de votre commune a-t-il plutôt augmenté ou diminué au cours des dix dernières années ? ॥ 
(46\%) et des charges financières y relatives (50.5\%), alors que la perception d'une centralisation est plutôt faible (respectivement $19.8 \%$ et $20.3 \%$ ). S'agissant des compétences stratégiques, la proportion s'élève à l'inverse à $16.6 \%$ pour un transfert du canton vers la commune et à $38.3 \%$ de la commune vers le canton ${ }^{14}$. Les communes se voient donc simultanément attribuer plus de responsabilités mais moins de ressources pour les assumer. La capacité décisionnelle, ou la possibilité de décider des tâches qu'il est nécessaire et prioritaire de mettre en œuvre pour répondre aux exigences dont les citoyens peuvent faire directement part au gouvernement local, est une composante cruciale de l'autonomie locale. Il n'est donc pas étonnant de constater que les secrétaires communaux ont, dans une très large majorité, décrété que les transferts de charges, de tâches et de compétences décisionnelles liées aux réformes ont globalement " pas du tout " conduit à un renforcement de l'autonomie communale (Figure 4). Selon les résultats de l'enquête, les réformes de répartition des tâches ont plutôt débouché sur un désenchevêtrement des tâches décisionnelles, opérationnelles et financières correspondantes (45.2\%), ainsi que sur la création de structures régionales de mise en œuvre (45.8\%). En outre, l'ambition prônée par la réforme de répartition des tâches de consulter davantage les communes - concrétisée notamment au travers de l'art. 19.2 de la Loi sur les Subventions (LSu, RO 616.6) - semble ne constituer qu'un effet d'annonce, dans la mesure où seules les villes de plus de 10'000 habitants considèrent à plus de $70 \%$ avoir une certaine influence sur le processus de décision relatif aux transferts de charges, de tâches et de compétences décisionnelles.

Figure 3 : Perception de l'évolution de l'autonomie communale de 1999 à 2009 en fonction de la population (en\%)

\begin{tabular}{|c|c|c|c|c|c|c|}
\hline & & $\begin{array}{c}\text { En } \\
\text { augmentation }\end{array}$ & Pas d'évolution & En diminution & Sans réponse & Total \\
\hline \multirow{5}{*}{ Communes (nb d'habitants) } & -499.00 & 4 & 24 & 68 & 5 & 314 \\
\hline & $500-999$ & 5 & 23 & 69 & 3 & 266 \\
\hline & $1000-4999$ & 6 & 24 & 68 & 2 & 620 \\
\hline & $5000-9999$ & 12 & 27 & 59 & 1 & 115 \\
\hline & $10000+$ & 10 & 28 & 63 & 0 & 80 \\
\hline Total & & 6 & 24 & 67 & 3 & 1395 \\
\hline
\end{tabular}

Source : enquête nationale sur les secrétaires communaux, IDHEAP et KPM, 2009, question $18 b$.

\footnotetext{
${ }^{14}$ Sur 574 retours, $29.2 \%$ des secrétaires communaux ont répondu « pas de transfert » de charges financières, $34.2 \%$ de tâches à mettre en œuvre et $45.1 \%$ de compétences stratégiques. Source : enquête nationale sur les secrétaires communaux, IDHEAP et KPM, 2009.
} 


\section{Figure 4 : Renforcement de l'autonomie communale au travers des réformes de répartition des} tâches en fonction des cantons

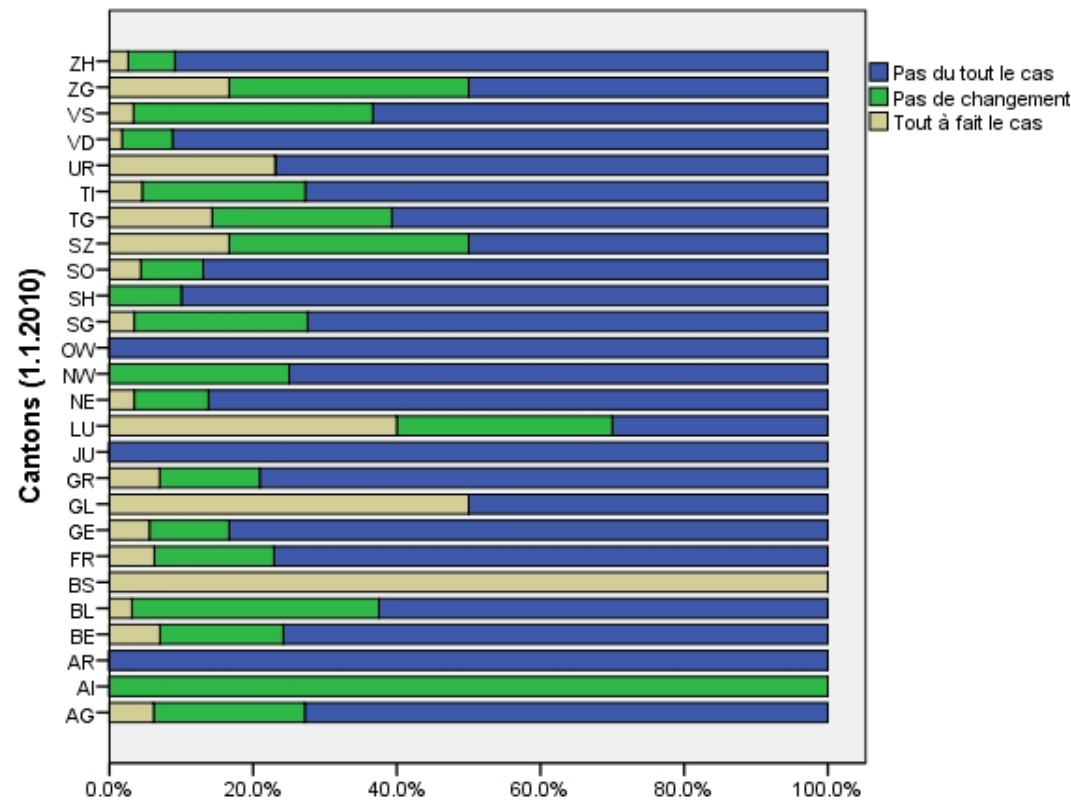

Source : enquête nationale sur les secrétaires communaux, 2009, question $22(N=937$; coefficient de contingence $=.414)$

\section{Discussion}

En Suisse, les niveaux d'autonomie cantonale et communale sont élevés en comparaison internationale. Ils s'expliquent par les différents évènements historiques qui ont progressivement constitué le système politique suisse (path dependency: Page, 2006). En effet, la Suisse s'inscrit dans un processus traditionnel: elle poursuit une évolution définie par les événements et les décisions prises au préalable. Les cantons et les communes ont, dès l'origine de la constitution helvétique, acquis une forte autonomie et un grand pouvoir de décision. Contrairement à bien d'autres pays européens, la Suisse s'est construite dans un processus bottom-up, soit de bas vers le haut; ce sont les cantons qui ont progressivement donné des compétences à la Confédération et non l'inverse, de même que les communes l'ont fait avec les cantons. Pour contrer cette tendance centralisatrice croissante face à la multiplication des tâches publiques et des coûts de mise en œuvre, le peuple suisse a voté la réforme de la répartition des tâches en 2008. La réforme a permis d'instaurer une collaboration verticale plus forte basée sur un partenariat non-hiérarchique et de diviser les responsabilités. Le désenchevêtrement a malgré tout atteint ses limites puisqu'il n'a pas été possible de le faire dans le cas de vingt-et-une tâches communes dont quinze sont traitées par les conventions-programmes. Même si l'évaluation de la réforme est aujourd'hui positive, il reste encore des problèmes de courts-terme à régler, tels que l'influence trop grande de la Confédération dans l'application de mise en œuvre, la fixation des objectifs dans les réglementations conjointes et le manque notable de communication entre cette dernière et les cantons.

L'incidence des réformes entre la Confédération et les cantons a poussé un grand nombre de cantons à lancer parallèlement des réformes de réallocation des tâches avec leurs communes ${ }^{15}$.Ces dernières ont été effectuées dans le même esprit que celles de l'échelon supérieur, soit dans une perspective générale de

\footnotetext{
${ }^{15}$ Mentionnons, à titre d'exemple les cantons suivants: Argovie (1996-2010), Berne (1994-1998), Neuchâtel (1994-2004) ; Nidwald (1996-1998); Obwald (1998-2001) ; Vaud (1996-2002) ; Jura (1996-2004) ; Lucerne (1997-2002) ; Schaffhouse (1997-2003) ; Zoug (1994-2005) ; Bâle-Ville (2004-2007) ; Schwyz (1998-2001). Ceux-ci profitaient souvent d'une adaptation de leur constitution suite à l'entrée en vigueur en 2000 de la nouvelle constitution fédérale.
} 
modernisation de l'Etat et dans la volonté d'améliorer l'efficacité allocutive et l'enchevêtrement des tâches. II est vrai que les enjeux étaient comparables entre les deux niveaux. Ainsi, il est intéressant de constater que les mêmes principes fondamentaux sont présents : la subsidiarité, l'équivalence, et celui du décideur-payeur. Trois différences fondamentales peuvent néanmoins être relevées (Jacot-Descombes, 2013) : premièrement, le type d'instrument des conventions-programmes conclus entre la Confédération et les cantons n'a que très peu été introduit au niveau inférieur ; deuxièmement, la Confédération et les cantons bénéficiaient d'une marge de manœuvre plus étendue pour revoir la répartition des tâches entre eux, dans les limites du droit national, alors que les cantons étaient tenus d'appliquer le droit fédéral qui exige de plus en plus de tâches cantonalisées; enfin, en rapport avec ce dernier point, les cantons, contrairement à la Confédération dans une certaine mesure, ont préféré une certaine centralisation des compétences pour éviter des mises en œuvre lacunaires des communes bénéficiant de moins de ressources.

Cette dernière dissemblance fait échos aux tendances se dessinant en termes d'impacts que provoque la mise en application des réformes sur les autonomies régionale et locale respectivement. En effet, l'indépendance des cantons vis-à-vis du pouvoir fédéral est grande à l'origine. Elle est de plus en voie de renforcement au travers de la réforme de la répartition des tâches puisque celle-ci ambitionne d'augmenter l'autonomie régionale en permettant aux cantons de participer davantage aux négociations et à l'élaboration des tâches publiques et à la prise de décision sur le terrain. Au niveau communal, la tendance générale consiste à un transfert de tâches. Mais, simultanément, la diminution de l'autonomie effective semble se maintenir au travers des réformes et au-delà par la restriction des ressources financières et des compétences décisionnelles. Il semblerait que les cantons, à qui l'on attribue la responsabilité de davantage de tâches, ont tendance à déléguer leur exécution à des agences semi-publiques ou privées ou aux communes, considérées de plus en plus comme des agents d'exécution comme des autres.

\section{Conclusion}

Bien que la RPT se soit déroulée il y a plus de six ans, il n'est pas encore possible de tirer des conclusions définitives en termes d'impacts sur l'autonomie cantonale et locale en Suisse. En effet, la grande variété des tâches publiques traitées par les conventions-programmes et les importantes disparités cantonales complexifient les évaluations mises en place par la Confédération et les cantons. Par exemple, il n'existe aucune grille de référence officielle émise par la Confédération concernant le nombre exact de conventionsprogrammes en vigueur et presque aucune évaluation cantonale sur les conséquences de la réforme au niveau des communes. Une thèse sur l'impact de la réforme de la répartition des tâches sur la collaboration entre la Confédération et les cantons à travers les conventions-programmes est d'ailleurs en cours à l'IDHEAP. Elle ambitionne de donner une vision plus large des conséquences de la réforme au niveau institutionnel et de contribuer à la réflexion qui découle de l'après-réforme.

Diverses recherches sont également prévues pour approfondir les connaissances des impacts des réformes au niveau local sur un plus long terme. Un questionnaire sera notamment adressé aux secrétaires communaux de l'ensemble des communes suisses en 2015. Parce que le système de répartition des tâches entre niveaux, constitutif de tout système fédéraliste, est en constante mutation. Suite à l'entrée de sa nouvelle constitution le 1er juin 2013, le canton de Genève a par exemple lancé en 2014 une large réforme de la répartition des tâches communes-canton, régie par les principes de " proximité, de subsidiarité, de transparence et d'efficacité " et qui vise notamment à " revaloriser le sens de l'autonomie communale ${ }^{16}$. II s'agit donc de voir comment se traduit concrètement cet objectif louable sur les différentes composantes de l'autonomie communale.

\footnotetext{
${ }^{16}$ http://ge.ch/communes-canton, consulté en janvier 2015.
} 
Ainsi, la réforme produit différents effets sur les niveaux institutionnels. S'ensuit un renforcement de la segmentation horizontale et verticale du fédéralisme suisse par l'attribution de tâches exclusives et conjointes aux institutions. Les tâches communes sont gérées par la Confédération et les cantons qui se consultent lors d'un processus de négociation et ces derniers, à leur tour, consultent les communes. Cette mixture de coopération, communication et négociation forme une véritable gouvernance multi-niveaux. Le paysage public suisse se transforme et s'élargi. 


\section{Bibliographie}

ARMINGEON, K. (2000): Swiss Federalism in Comparative Perspective. In : Wachendorfer-Schmidt, U. (ed) : Federalism and Policy Performance. London/New York : Routledge, 112-129

ATHIAS Laure, (2013) : "La contractualisation du service public: une analyse économique”, Chapitre 34. Dans: Manuel d'administration politique Suisse, Presses polytechniques et universitaires romandes

BOCHSLER, Daniel, KOLLER Christophe, SCIARINI Pascal, TRAIMOND Sylvie, TRIPOLINI Ivar, (2004): Les cantons suisses sous la loupe. Dans : Autorités, employés publics, finances. Berne: Haupt.

CAPPELLETTI, Fabio, FISCHER, Manuel \& SCIARINI, Pascal (2014): « Let's Talk Cash: Cantons' Interests and the Reform of Swiss Federalism", In: Regional \& Federal Studies, 24:1, 1-20

CENI, Monique (2008): « Guerre, impôts fédéraux directs et fédéralisme d’exécution » Dans:

Schweizerische Gesellschaft für Wirtschafts - und Sozialgeschichte, Zürich

CHEVALLIER, Jacques, (2008): « Contractualisation et régulation », Dans : Chassagnard-Pinet, D. Hiez (dir.), La contractualisation de la production normative, Dalloz, Coll. Thèmes et commentaires, 2008, pp. 83-93

CLARK, Gordon L. (1984): A Theory of Local Autonomy. In: Annals of the Association of American Geographers 74(2): 195-208.

CONSEIL DE L'EUROPE (1985): Charte européenne de l'autonomie locale. http://conventions.coe.int/Treaty/en/Treaties/Html/122.htm (consulté en janvier 2015).

CONSEIL FEDERAL (2003): Message relatif à la Charte européenne de l'autonomie locale du 19 décembre 2003. Bern. 03.079.

DAFFLON, Bernard, (2004): « Le désenchevêtrement des tâches et la péréquation financière : les chantiers du fédéralisme suisse après le 28 novembre ", Chaire de finances publiques, Faculté des Sciences économiques et sociales, Université de Fribourg

EMERY, Yves, (2000) : «L'administration suisse, condamnée à se moderniser », IDHEAP

FLEINER Thomas (2002): "Recent developments of Swiss federalism", In: Publius, Nr. 2, S. 97- 123.

FREIBURGHAUS, Dieter (2005): „Bedingungen für eine gelingende Föderalismusreform: Die Neugestaltung des Finanzausgleichs und der Aufgabenteilung zwischen Bund und Kantonen in der Schweiz", Jahrbuch des Föderalismus 6: 506-515.

FREY, René L., Georg KREIS, Gian-Reto PLATTNER, René RHINOW, (2005): « Le fédéralisme suisse : la réforme engagée. Ce qui reste à faire ", Le savoir suisse, PPUR, p. 17

GESER, Hans (1997): Zwischen Aufgabenzuwachs und Autonomieverlust: Neue Selbstbehauptungsstrategien der Gemeinden im Zeitalter "vertikaler Politikverflechtung". In: Zürich: Soziologisches Institut der Universität Zürich. http://socio.ch/gem/t_hgeser5.htm

GIAUQUE, David et EMERY, Yves (2008) : Repenser la gestion publique ; bilan et perspectives en Suisse, Le savoir Suisse, PPUR, p. 40-41

GOLDSMITH, Michael and Edward C. PAGE (2010): Changing government relations in Europe: from localism to intergovernmentalism. London: Routledge. 
GURR, Ted Robert and KING, Desmond S. (1987): The State and the City. London: Macmillan.

HANSEN, Tore and Jan E. KLAUSEN (2010): Between the Welfare State and Local government Autonomy. In: Local government Studies 28(4): 47-66.

HESSE, Joachim Jens and Laurence J. SHARPE (1991): Local Government in International Perspective: Some Comparative Observations. In: Joachim Jens Hesse (Ed.): Local government and Urban Affairs in International Perspective. Baden-Baden: Nomos, 603-621

HOOGHE L. \& MARKS G. (2001): Multi-level governance, New York, Rowan and Littlefield HORBER-PAPAZIAN, Katia (2006): „Les communes“. In KLÖTI et al. Manuel de la politique suisse (pp. 233-258). NZZ

JACOT-DESCOMBES, Caroline (2013): A la croisée des modèles du fédéralisme fiscal et coopératif: les résultats des réformes cantons-communes en Suisse. Thèse de doctorat. UNIL/IDHEAP.

KNAPP, Blaise (1986) : "Etapes du fédéralisme suisse", Germann, R. E. und E. Weibel (Hrsg). Dans: Manuel Système politique de la Suisse, Vol. 3. Bern: Haupt. S. 31-54.

KNOEPFEL Peter, LARRUE Corinne et VARONE Frédéric (2006) : Analyse et pilotage des politiques publiques, Zürich, Chur, Ruëgger Verlag, 2e éd.

LADNER, Andreas, KEUFFER, Nicolas (2014): Local autonomy, a multidimensional concept: How to measure local autonomy, how to explain different degrees of local autonomy and what about its effects? Paper presented at the 2014 EGPA Annual Conference in Speyer (Germany), 10-12 September 2014.

LADNER, Andreas (2013) : „Etat, système politique et accomplissement des tâches“, dans: Ladner, Andreas, Jean-Loup Chappelet, Yves Emery, Peter Knoepfel, Luzius Mader, Nils Soguel und Frédéric Varone (Eds) (2013). Manuel d'administration publique en Suisse. Lausanne: PPUR. p. 7-30.

LADNER Andreas, CHAPPELET Jean-Loup and EMERY Yves, KNOEPFELPeter and MADER Lucius \& SOGUEL Nils and VARONE Frédéric (2013): Manuel d'administration publique Suisse, PPUR

LADNER, Andreas (2005): Switzerland: Reforming small autonomous municipalities. In: Comparing Local Governance: Trends and Developments. Denters, Bas and Rose, Lawrence (eds). Basingstoke/New-York: Palgrave Macmillan, 139-154.

LADNER, Andreas, ARN, Daniel, FRIEDRICH, Ueli, STEINER, Reto, WICHTERMANN, Jürg (2000): Gemeindereformen zwischen Handlungsfähigkeit und Legitimation. Bern: Institut für Politikwissenschaft und Institut für Organisation und Personal.

LADNER, Andreas (1994): Finanzkompetenz in der Gemeinde - Ein Überblick über die Praxis. In: Finanzen der öffentlichen Hand Nr. 5 "Finanzföderalismus". Bern: Emissionszentrale der Schweizer Gemeinden, 65-85.

LERESCHE, Jean-Philippe (1996) : Les nouvelles échelles de gestion urbaine : entre conflit et coopération. Jaccoud, Christophe, Martin Schuler et Michel Bassand (éds). Dans: Raisons et déraison de la ville. Approches du champ urbain. Lausanne : PPUR, 255-274.

LINDER, Wolf, (1987) : La décision politique en Suisse : genèse et mise en œuvre de la législation, Dans: réalités sociales, Lausanne, écrit suite à la PNS ${ }^{\circ} 6$ 
OFS - Office fédéral des statistiques (2014): Amtliches Gemeindeverzeichnis der Schweiz Angekündigte Änderung 2014 - Ausgabe von 02.10.2014. http://www.bfs.admin.ch/bfs/portal/en/index.html

PAGE Scott E., (2006), Path Dependency, In: Quaterly Journal of Public Administration, 1 :87-115 PAGE, Edward and Michael GOLDSMITH (1987): Central and Local government Relations. London: Sage. POLLITT C, BOUCKAERT G. (2004) : Public Management Reform, A comparative Analysis, Oxford

SCHARPF, Fritz W. (1978) : Die Theorie der Politikverflechtung: ein kurzgefasster Leitfaden, In: Hesse, J. J. (ed.) Politikverflechtung im föderativen Staat. Studien zum Planungs- und Finanzierungsverbund zwischen Bund, Ländern und Gemeinden, Baden-Baden, Germany: Nomos.

STEINER, Reto et LADNER, Andreas (2006) : Die Schweizer Gemeinden im Fokus - Ergebnisse der Gemeindebefragung 2005. Glatthard, Alexander/Isch, Ulrich (Hrsg). In: Perspektiven für Gemeindefinanzen, Bern, S. 8-35.

VATTER, Adrian (2006): Föderalismus. in: Klöti, Ulrich et al. (Hrsg.). Handbuch der Schweizer Politik. Zürich: NZZ. 4. Auflage. S. 79-102.

VERHOEST, K., PETERS, B. G., BOUCKAERT, G. \& VERSCHUERE, B. (2004): The study of organisational autonomy: a conceptual review, In: Public Administration and Development, 24 (2), pp.101-118.

WALTI Sonja, (2001) : Le fédéralisme d'exécution sous pression ; la mise en œuvre des politiques à incidence spatiale dans le système fédéral suisse, Helbong et Lichtenhahn, IDHEAP, GE, BL, Munich

WELLSTEIN, Gérard, (2001) : "Analyse de la RPT entre la Confédération et les cantons », Dans: La Vie économique, Revue de politique économique

\section{Rapports relatifs à la RPT}

"Monitoring du fédéralisme 2011-2013 » de la Fondation CH pour la collaboration confédérale publié le 20 juin 2014

« Message sur la législation d'exécution concernant la réforme de la péréquation financière et de la répartition des tâches entre la Confédération et les cantons » du Conseil fédéral, publié le 7 septembre 2005, n05-070

« Rapport sur l'évaluation de l'efficacité de la RPT entre la Confédération et les cantons " publié par le Conseil fédéral et réalisé par l'AFF, mars 2014

« Rapport sur l'évaluation de l'efficacité de la RPT : objectifs atteints, mais débat nécessaire - dossier politique » par Economiesuisse, publié le 5 mai 2014

"Message sur la Réforme de la péréquation financière et de la répartition des tâches entre la Confédération et les cantons » du Conseil fédéral, publié le 14 novembre 2001, n05-074

"Risques et défis liés aux Conventions-programmes ; rapport de synthèse ", rédigé par le CDF, publié le 2 avril 2014, $n^{\circ} 1.12507 .601 .00402 .01$

«Préparatifs et pierres d'angle », Thème clé de la RPT 3, publié en septembre 2004 par le DFF et la CdC 